\title{
The use of nanotechnology in cardiovascular disease
}

\author{
Meera Chandarana ${ }^{1} \cdot$ Anthony Curtis ${ }^{1} \cdot$ Clare Hoskins $^{1}$ (D)
}

Received: 3 May 2018 / Accepted: 10 August 2018 / Published online: 16 August 2018

(c) The Author(s) 2018

\begin{abstract}
Cardiovascular diseases claim a number of lives globally; many of which are preventable. With the increase in diets that consist of high saturated fat, salt, and sugar, people often living sedentary lifestyles, and a rise in cases of obesity, the incidence of cardiovascular disease is increasing. These contributing factors, coupled with more advanced methods of diagnosis, have delivered statistics that clearly show that there is a rising trend in the prevalence of cardiovascular disease. Treatment for cardiovascular diseases is limited currently to oral medicines or invasive surgery. There is a huge gap in this area of medicine for novel therapeutics for improved patient outcomes. Nanotechnology may provide a solution to more effective treatment of disease, with better prognoses and a reduced side effect profile. This review will explore for potential solutions to the limited pharmacological therapies currently on the market and the future that lies ahead for the place of nanotechnology within cardiovascular medicine.
\end{abstract}

Keywords Nanomedicine $\cdot$ Cardiovascular disease $\cdot$ Nanotechnology $\cdot$ Medical therapeutics

\section{Introduction}

Cardiovascular diseases (CVDs) are the leading cause of death worldwide, claiming 17.7 million lives in 2015 , and this figure is projected to increase to 23.6 million in 2030 (WHO 2017). The term 'cardiovascular diseases' encompasses, but is not limited to coronary heart disease, deep vein thrombosis, pulmonary embolism, and myocardial infarction (MI), all of which result in ischaemia and tissue death. Of these, MI and heart failure are the major conditions which lead to mortality.

A MI, known commonly as a heart attack, is the result of total occlusion of the major arteries supplying the heart leading to a decrease in blood flow, oxygen, and nutrient supply. Ultimately, this results in myocardial tissue death by apoptosis or necrosis due to coronary occlusion. An MI can manifest as two different forms, ST-elevation MI (STEMI) or non-ST-elevation MI (NSTEMI), dependant on the visible changes on an ECG trace (NICE 2013). The current treatment for MI is reperfusion in the shortest period of time following arterial obstruction, which can be achieved

Clare Hoskins

c.hoskins@keele.ac.uk

1 School of Pharmacy, Institute of Science and Technology for Medicine, Keele University, Keele ST5 5BG, UK through invasive methods such as percutaneous coronary intervention (PCI) or coronary artery bypass graft (CABG), or through pharmacological therapy (Ruiz-Esparza et al. 2013).

Heart failure is a clinical disease state that presents with symptoms of breathlessness, peripheral oedema, and fatigue, all of which are a result of reduced cardiac functionality (Ponikowski et al. 2016). This form of cardiomyopathy is a state in which the heart muscle is unable to pump efficiently to satisfy the body's metabolic requirements (Ruiz-Esparza et al. 2013). Disease of the coronary artery is the largest contributory factor in the development of heart failure (Fox et al. 2001). Heart failure is an umbrella term that refers to a range of various cardiac syndromes, including: left-ventricular dysfunction, valvular, pericardial, or endocardial abnormalities, and irregularity of rhythm (Ponikowski et al. 2016). It is estimated that up to 2 million people are living with heart failure in the UK, a figure that is continuously increasing due primarily to the significantly aging population (Mitchell et al. 2015). Currently, guidance recommends ACE inhibitors and $\beta$-receptor blockers as the first-line treatment for heart failure (NICE 2010).

The current statistics regarding the incidence of CVDs highlight the need for new therapies and technological advancements to combat these diseases. 

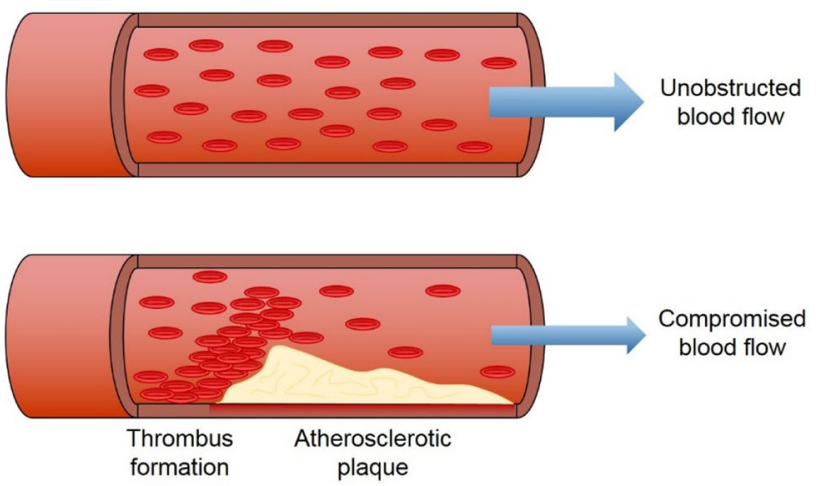

Fig. 1 Progression of atherosclerotic plaque showing narrowing of the blood vessel

Atherosclerosis is the precursor to several vascular diseases. Atherosclerotic plaques are formed of macrophage cells, fat, cholesterol, and calcium, which become deposited in the vasculature and the growing plaques harden over time. This results in narrowing of the blood vessels, a reduction in blood flow, rupture, and ultimately ischaemia ((Weaver 2013) (Fig. 1). Histologically, the rupture of plaques, which leads to a cardiovascular event, is a result of poor fibrous plaque caps containing increased concentrations of matrix metalloproteinases (MMPs) that cause weakness in the plaque (Qin et al. 2016). Lifestyle factors are eminent contributors to the development of atheromatous plaques. It is widely accepted that consuming foods rich in salt, sugar, and saturated fat greatly increases the risk of suffering from a CVD in later life. Having a high low-density lipoprotein (LDL)-to-high-density lipoprotein (HDL) ratio is also a risk factor as LDLs are deposited on the walls of blood vessels and contribute to the development of plaques. Hypertension, smoking, lack of physical activity, family history, obesity, age, and stress all play a role in the development of CVDs, estimated to cost the economy $£ 19$ billion per annum, with the National Health Service (NHS) bearing a burden of $£ 9$ billion (British Heart Foundation 2017).

Current treatments for CVDs are focussed on restoring normal blood flow through or around the damaged vasculature and the prevention of recurrent cardiovascular insults. Reduction in further build up and thickening of atherosclerotic plaques, as well as effects on external elastic membranes and fibrous and dense calcium volumes is achieved by statin therapy (Banach et al. 2015). Dual antiplatelet therapies using cyclooxygenase inhibitors such as aspirin and P2Y12 inhibitor such as clopidogrel are first-line treatments for prevention of CVDs, which aim to reduce clot formation and platelet aggregation (Kapil et al. 2017). There is a requirement for improvement in these treatments, primarily due to the risks associated with taking antiplatelet therapy which significantly adverse side effect profiles, and poor patient compliance (Harrington et al. 2003). In addition to this, some patients do not respond well to antiplatelet therapy, which has a negative effect on their long-term prognosis. Studies have demonstrated that patients who have suffered an acute myocardial infarction but have a low response to clopidogrel are at increased risk of recurrent cardiovascular events at follow-up (Geisler et al. 2006). This supports the necessity for advancements in technology and an opportunity for nanomedicine (Fig. 2).

\section{Nanotechnologies for treatment}

The field of nanomedicine comprises the diagnosis, treatment, and prevention of disease or injury to improve and maintain a good quality of life and health, with use of nanotechnology (Patil et al. 2008). For example, a domain of nanomedicine aims to control and manipulate biomacromolecular and supramolecular entities which

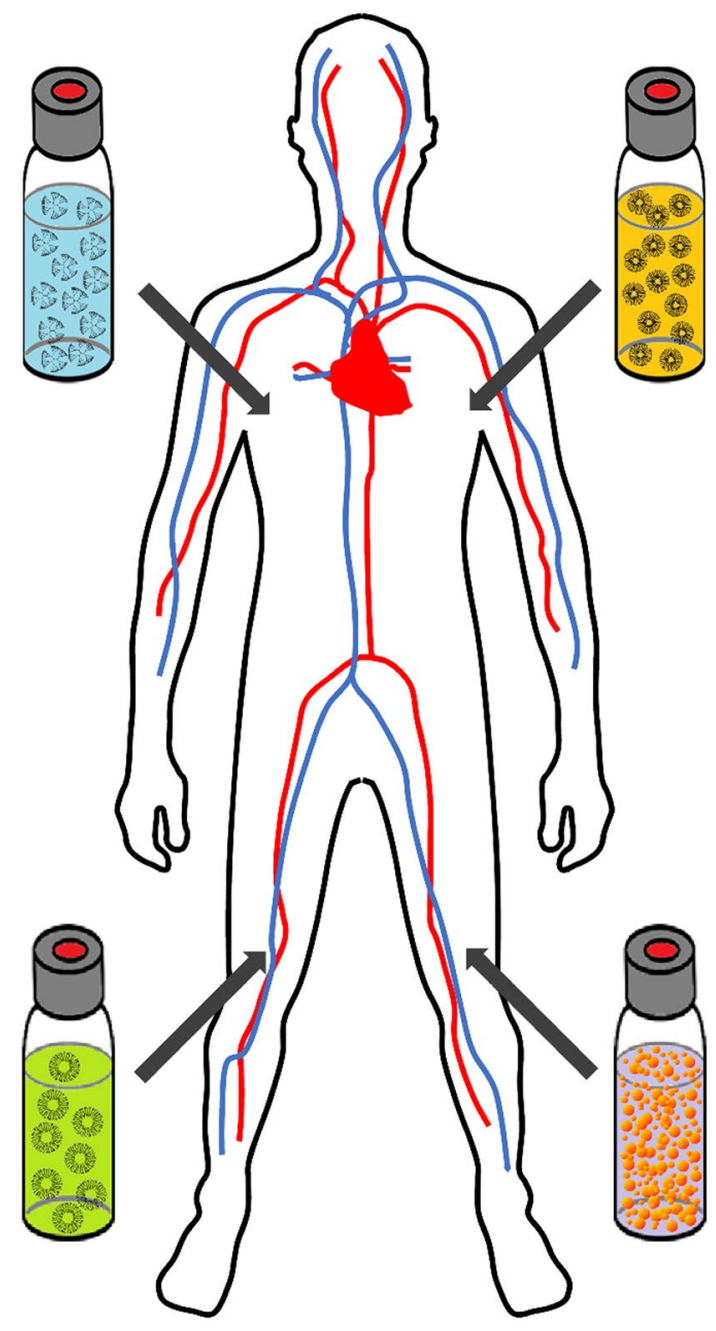

Fig. 2 Use of nanotechnology for cardiovascular disease 
are vital to human health, encompassing DNA, RNA, cell membranes, and lipid bilayers (Tomalia et al. 2007). Nanoparticles, in particular, are widely accepted as having physicochemical properties that improve biological function, such as a high surface energy due to their large surface area to volume ratio, wettability, reactivity, and roughness (Jiang et al. 2017). The British Standards Institute defines nanoparticles as microscopic particles with all three dimensions in the nanoscale, ranging from 1 to $100 \mathrm{~nm}$ (British Standards Institute 2011). Advancements in nanomedicine research have led to better ways to reduce toxicity, prolong the half-life of drugs, and reduce side effects through alteration of nanoparticle properties, whilst maintaining the particle biocompatibility (Gupta and Gupta 2005). Targeted drug delivery in treatment of disease using nanoparticles is possible through active or passive means: active targeting requires conjugation of the therapeutic agent to a tissue- or cell-specific ligand, whereas passive targeting involves coupling of the therapeutic agent to a high-molecular-weight polymer that has enhanced permeation and retention to vascular tissue (Patel and Bailey 2007).

A diverse range of nanotechnologies have been developed for biomedicine within the past 2 decades, each with their own unique properties and advantages. These include micelles, liposomes, dendrimers, nanoparticles, and the use of nano-coatings (De Jong and Borm 2008) (Fig. 3). Currently, therapeutic applications of nanotechnology within medicine are focused around cancer treatment and diagnosis (Sanna et al. 2014); however, the focus is shifting to other areas of therapeutics, such as cardiovascular medicine (Binsalamah et al. 2012) and antimicrobial resistance (Kumar et al. 2018). Table 1 summarises the main classes of technologies under exploration in this area.

\section{Liposomes}

Liposomes form phospholipid bilayer structures, around 50-200 nm in size, with aqueous cores encapsulated within natural phospholipids, such as cholesterol (Akbarzadeh et al. 2013). As they possess qualities of both hydrophilicity and hydrophobicity, liposomes can be used as effective drug delivery systems. There are three classifications of liposomes, based on their size and number of bilayers. Variable size, method of preparation, surface charge, and lipid composition are all contributing factors to the properties displayed by a liposome (Sahoo and Labhasetwar 2003). Small unilamellar vesicles (SUV) have a single lipid bilayer; large unilamellar vesicles (LUV) are similar to SUVs, whereas multilamellar vesicles (MLV) have numerous bilayers (Sahoo and Labhasetwar 2003). Manipulation of the surface of liposomes can be undertaken via the addition of poly(ethylene glycol) (PEG), giving the carrier 'stealth'-like properties (Immordino et al. 2006). The 'stealth liposomes' have a prolonged circulation time, reduced uptake, and reduced clearance by phagocytes or the liver (Sahoo and Labhasetwar 2003). Furthermore, antibodies or other targeting moieties can be attached to the liposomal surface to target specific areas (Levchenko et al. 2012). Due to the processes of platelet aggregation in myocardial infarction, atherosclerosis, and thrombosis, platelet-targeted liposomal drug delivery may prove to have potential therapeutic applications (Ruiz-Esparza et al. 2013). In regard to applications in cardiovascular medicine, liposomes have the potential to be utilised to treat peripheral artery disease and intermitted claudication. Currently, research into the liposomal drug delivery of prostaglandin E-1 (PGE-1), under the trade name Liprostin, is undergoing phase III clinical trials (Bulbake et al. 2017). PGE-1 possesses a wide range of pharmacological properties, including vasodilation, inhibition of platelet aggregation and leukocyte adhesion, and anti-inflammatory
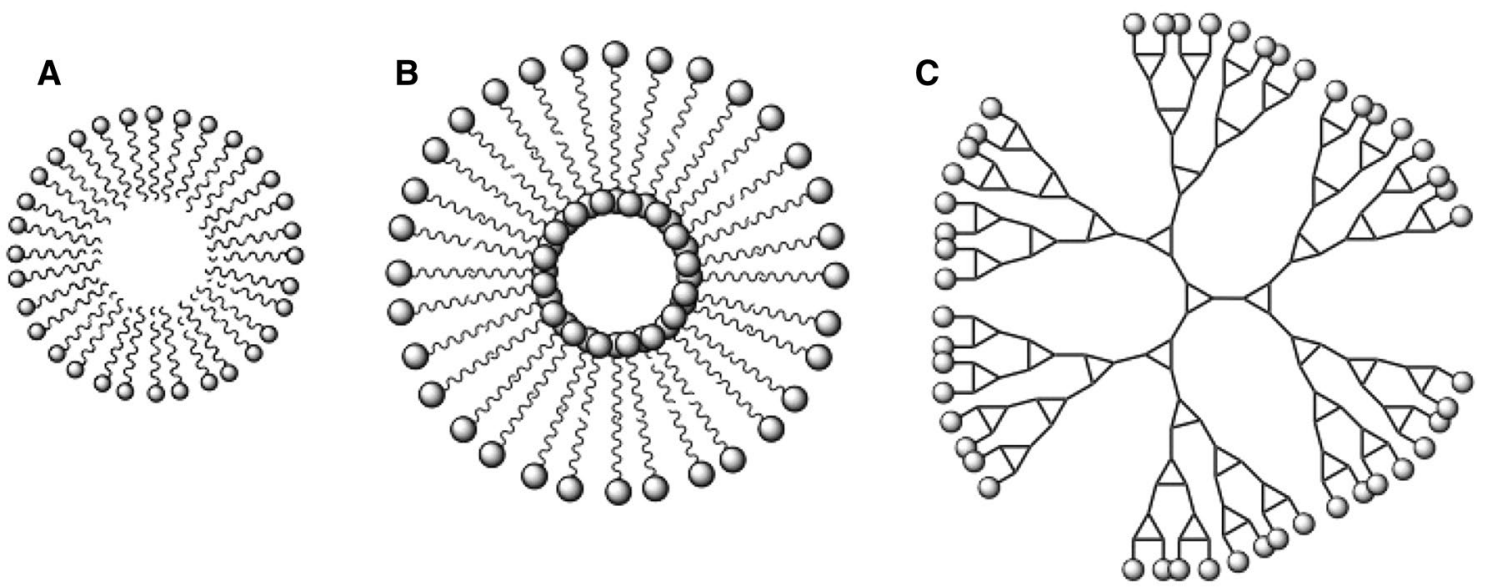

Fig. 3 Nanocarriers for the delivery of therapeutic agents: a micelle, $\mathbf{b}$ liposome, and $\mathbf{c}$ dendrimer

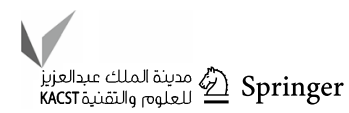


Table 1 Summary of nanotechnology applications in cardiovascular disease

\begin{tabular}{|c|c|c|}
\hline Class & Application & References \\
\hline \multirow[t]{4}{*}{ Liposome } & Thrombosis & Ruiz-Esparza et al. (2013), Zhang et al. (2018) and Palekar et al. (2013a, b) \\
\hline & Atherosclerosis & $\begin{array}{l}\text { Ruiz-Esparza et al. (2013), Chono et al. (2005), Alaarg et al. (2015) and Hosseini et al. } \\
\text { (2015) }\end{array}$ \\
\hline & Vasodilation & Bulbake et al. (2017), Jain et al. (2014) and Nahar et al. (2014) \\
\hline & Cardio-protectant & Zhang et al. (2014), Allijn et al. (2017) and Murohara et al. (1995) \\
\hline \multirow[t]{3}{*}{ Polymeric nanoparticle } & Thrombosis & Al Meslmani et al. (2017), Korin et al. (2012) and Pazzini et al. (2015) \\
\hline & Atherosclerosis & Matoba et al. (2017) and Sanchez-Gaytan et al. (2015) \\
\hline & Cardio-protectant & Giannouli et al. (2018) and Aisha et al. (2015) \\
\hline \multirow[t]{4}{*}{ Polymeric micelle } & Thrombosis & Pan et al. (2018), Chan et al. (2016) and Pang et al. (2014) \\
\hline & Atherosclerosis & Wu et al. (2018), Kuo et al. (2014) and Fares et al. (2018) \\
\hline & Vasodilation & El-Gendy et al. (2017), Önyüksel et al. (1999) and Zhai et al. (2013) \\
\hline & Cardio-protectant & Cote et al. (2015) and Siddalingappa et al. (2015) \\
\hline \multirow[t]{4}{*}{ Dendrimer } & Thrombosis & Zhang et al. (2017), Durán-Lara et al. (2013) and Mukhametova et al. (2017) \\
\hline & Atherosclerosis & Napoli et al. (2015) \\
\hline & Vasodilation & Singh et al. (2017) \\
\hline & Cardio-protectant & Wan et al. (2011) \\
\hline Gelated nanoparticle & Cardio-protectant & Oh et al. (2010) \\
\hline Nano-coating & Biocompatibility of implants & $\begin{array}{l}\text { Tan et al. (2013), Kidane et al. (2009), Finn et al. (2005), Costa et al. (2016), CeloNova } \\
\text { Biosciences (2018) and Acharya et al. (2012) }\end{array}$ \\
\hline Diagnosis & Imaging & $\begin{array}{l}\text { Kim et al. (1996), Thakor et al. (2011), Varna et al. (2018), Chen et al. (2014), Cai et al } \\
\text { (2005) and Palekar et al. (2015) }\end{array}$ \\
\hline
\end{tabular}

qualities (Gao et al. 2010). Encapsulation of PGE-1 within lipid nanoparticles reduced the degradation of PGE-1 and improved both the prolongation of the drug outcome and the anti-inflammatory effect (Gao et al. 2010), exhibiting real potential for PGE-1-loaded lipid nanoparticles in clinical application.

Liposomes have been designed for targeted treatment of thrombosis. Thrombosis, a blockage of the blood vessels, is associated with myocardial infarction (and stroke). Recently, liposomes carrying thrombolytic drug urokinase were reported; these were surface functionalised with a cyclic arginyl-glycyl-aspartic acid (cRGD) peptide (Zhang et al. 2018). cRGD peptides selectively bind onto the GPIIb/IIIa receptors on the active platelets conferring targeted delivery. In the cRGD, liposomes demonstrated similar effect to the free drug at $75 \%$ less drug dosage in a mouse mesenteric thrombosis model (Zhang et al. 2018).

Multiple studies have reported the encapsulation and delivery of berberine (Allijn et al. 2017; Lou et al. 2013; Sailor 2015). Berberine is a small fluorescent isoquinoline quaternary alkaloid which has proven to be useful at high doses as a cardio-protectant (Zhang et al. 2014) and for improvement of cardiac function (Huang et al. 2015). Berberine-loaded liposomes have been developed and tested for IL-6 secretion inhibition and cardiac function protection against adverse remodelling after $\mathrm{MI}$ in C57BL/6J mice (Allijn et al. 2017). In vivo, the liposomal berberine formulation significantly preserved cardiac ejection fraction 28 days
post-MI by $64 \%$ compared with the unloaded liposomes and free drug. This study highlights the potential which liposomal berberine formulation possess as a potential treatment of adverse remodelling after MI.

The use of liposomes is relatively old compared to the polymer-based systems and it is likely that these systems will be clinically applicable at an earlier stage. However, as observed in other liposomal drug delivery applications, there are some disadvantages. These include the lack of long-term stability of these systems resulting in premature drug release, as well. In addition, increased excipient-todrug ratios are often required making them more expensive and less effective than some of the polymer-based systems.

\section{Polymeric nanoparticles}

Polymeric nanoparticles have attracted particular interest in the nanomedicine field, due to the manipulative qualities of the materials and the potential of reabsorption in the body (Jiang et al. 2017). The route of delivery of polymeric nanoparticles is dependent on their site of action and the most minimally invasive method of getting them into systemic circulation. Intravenous injection is the most common method of administration; however, oral, mucosal, dermal, or transdermal administration is also viable (Elsabahy and Wooley 2012). Polymeric nanoparticles make up a large class of systems. These include solid nanoparticles, amphiphilic nanoparticles (forming micelles, vesicles, and 
rods), dendrimers, and star-shaped systems. All of these systems possess their own unique architecture and properties (Hoskins et al. 2012). In general, polymer-based systems are more cost-effective and easier to manufacture and scale up compared to liposomes with longer stability profiles.

There are a number of non-biodegradable polymers such as poly(methyl methacrylate) (PMMA), poly(acrylamide), poly(styrene), and poly(acrylates), (Banik et al. 2016); however, chronic toxicity has been observed in studies using these materials. This has led a focus on biodegradable polymers (Burman et al. 2015), examples of which include synthetic polymers such as poly(lactide) (PLA), poly(lactide-coglycolide) copolymers (PLGA), poly ( $\varepsilon$-caprolactone) (PCL), and 17 and poly(amino acids) (Elsabahy and Wooley 2012).

Expanded polytetrafluoroethylene (ePTFE) grafts are most commonly used to substitute blood vessels that have been damaged as a result of ischaemia or atherosclerotic plaques, re-establishing regular blood flow. A study was conducted using poly(lactic-co-glycolic acid) (PLGA) nanoparticles covalently bonded to ePTFE surfaces to test their therapeutic effects. Following oxidation of the ePTFE surface using $\mathrm{H}_{2} \mathrm{O}_{2} / \mathrm{H}_{2} \mathrm{SO}_{4}$, aminolyzation using 3-aminopropyl triethoxysilane was undertaken. The nanoparticles showed good stability on the ePTFE surface and the biocompatibility of the graft with mouse fibroblasts which demonstrated no substantial cytotoxicity (Al Meslmani et al. 2017), suggesting promise for the use of ePTFE for the treatment of CVD by incorporating antithrombotic drugs to the polymer grafts. Korin et al. (2012) studied the effects of biodegradable poly(lactic-co-glycolic) acid (PLGA) nanoparticles laden with the enzyme tissue plasminogen activator (tPA) which aids in the dispersion of clots. The nanoparticles were designed specifically to disintegrate and release the drug upon encountering high shear stress resulting from the narrowing of the surrounding vasculature (Korin et al. 2012). In in vivo studies, the complexes released tPA rapidly, thus dissolving the clot swiftly, which increased the survival rate following an induced pulmonary embolism (Korin et al. 2012). Furthermore, the results indicated that lower doses of tPA were required if it was incorporated in the polymeric grafts, suggesting a reduced side effect profile. This research clearly indicates that there are applications for this technology in the treatment of CVDs with the potential to transform cardiovascular medicine.

Quercetin $(\mathrm{Qu})$ encapsulated within polymeric nanoparticles (Giannouli et al. 2018) or as solid drug nanoparticles (Kakran et al. 2012; Tefas et al. 2015) has been the subject of a number of investigations. Qu is an antioxidant drug which has shown to protect against CVDs. One recent study reported a novel poly(lactic-co-glycolic acid) (PLGA) loaded with $\mathrm{Qu}$ for atherosclerosis prevention (Giannouli et al. 2018). The particles showed promising encapsulation efficiencies and drug release profiles indicating these systems as possible formulations to protect against CVDs (Giannouli et al. 2018). Work is ongoing to further explore the in vitro and in vivo potential of these particles.

\section{Micelles}

Micelles are amphiphilic structures with characteristic hydrophobic cores and hydrophilic shells, fashioned from either polymer- or lipid-based amphiphilic molecules (Gupta 2011). They form spontaneously due to a reduction in free energy arising from aggregation of the hydrophobic portions from the aqueous surrounding, and the creation of a micelle core stabilised with hydrophilic fragments exposed to the water (Mourya et al. 2011). As the concentration of an amphiphile increases in solution, an increase in the free energy of the system occurs due to the unfavourable conditions of the environment, as a result of interactions between water and the hydrophobic section of the amphiphile, which results in a decrease in the system entropy and structuring of the water in solution (Letchford and Burt 2007). Micelles are formed as the critical micelle concentration (CMC) is reached (Trinh et al. 2017). The formation of micelles at the CMC lessens the number of undesirable interactions with water as the hydrophobic moieties are effectively removed from the aqueous environment. The hydrophobic nature of the core allows for encapsulation of drugs, and the hydrophilic shell increases circulation time and systemic exposure to the micelle. Their small size and physicochemical properties allow for entry into tissues by crossing membranes (Eniola-Adefeso et al. 2012). Polymeric nanoparticles possess increased stability compared to low-molecular-weight surfactants and liposomes; this is due to their extremely low excipient:drug ratios. Hence, there is reduced chance of systemic toxicity resulting from the carrier vehicle but also makes them more cost-effective. The synthesis is highly controllable, and they can easily be tailor made to application, i.e., addition of targeting ligands or tracking molecules. Most commonly, the polymers used for micellar drug delivery are comprised of polyester or poly(amino acid) derivatives to form the hydrophobic region (Gaucher et al. 2005). Forms of polyester that are approved for use in human applications include poly(lactic acid) (PLA), poly( $\varepsilon$-caprolactone) (PCL), and poly (glycolic acid) due to their biocompatibility, biodegradability, and structural properties (Gaucher et al. 2005). Reports on the use of polymeric micelles for cardiovascular disease are rapidly emerging in the literature (Ma et al. 2017; Chmielowski et al. 2017; Singla et al. 2018; Wu et al. 2018).

An in vitro study demonstrated successfully the use of a block co-polymer micelle formed from poly(ethylene glycol) (PEG)-block-polycation carrying a (PEG- $b$-P[Asp(DET)]) side chain as a gene delivery vector for treatment of vascular disease, with efficient gene expression and low cytotoxicity in vascular smooth muscle cells (Akagi et al. 2007). To 
fully understand the in vivo outcome of gene transfer, the PEG- $b$-P[Asp(DET)] micellar formulation was implanted to a rabbit carotid artery and the degree of gene expression of plasmid DNA (pDNA) to the vascular region was determined. The carotid was wounded using a balloon catheter, inducing neointimal hyperplasia, and the encapsulated pDNA was administered. The presence of pDNA was detected using the FLAG sequence (p-MP-FLAG). The artery was stained with an anti-FLAG antibody to confirm the expression of the gene, the results of which confirmed that the micellar formulation was superior in comparison to the controls, homopolymer P[Asp(DET)], and branched polyethyleneimine (BPEI). The control vectors displayed a significant vessel occlusion by thrombus, which the micellar vectors studied did not exhibit (Akagi et al. 2007). The results of this study show that limitations with the current technology which uses viral vectors, such as antigenicity and oncogenicity, directs research towards use of non-viral vectors, since the safety and efficacy profiles of nanomicelles are superior (Kagaya et al. 2011).

Recently, an intelligent delivery system which responds to the oxidative atherosclerotic plaque microenvironment was reported (Wu et al. 2018). Block copolymer micelles were constructed using poly(ethylene glycol) and poly(propylene sulphide) (PEG-PPS). The micelles were used to solubilise andrographolide to decrease inflammatory response and the level of reactive oxygen species (ROS) for atherosclerosis treatment. Andrographolide is a plant-derived compound which exhibits cardiovascular protection effects via downregulation of inducible nitric oxide synthase expression and upregulation of endothelial nitric oxide synthase expression (Kajal et al. 2016). The stimuli responsive micelles formed resulted in rapid drug release triggered by the ROS, but also consumed the ROS by itself at the pathologic sites, resulting in the suppression of the pro-inflammatory cytokines ( $\mathrm{Wu}$ et al. 2018). The authors concluded that the novel formulation demonstrated excellent therapeutic effects both in vitro and in vivo, and hence, it may have promising potential against atherosclerosis (Wu et al. 2018).

Cationic-mixed polymeric micelles have been developed for targeted delivery of lumbrokinase (LK) for the treatment of thrombosis (Pan et al. 2018). LK is a fibrinolytic enzyme originated from earthworm which has shown to possess antithrombotic action (Wang et al. 2013). The micelles consisted of a block copolymer of polycaprolactone- $b$-poly (2(dimethylamino) ethyl methacrylate) (PCL-PDMAEMA), as well as a block copolymer of methoxy polyethylene glycol- $b$-polycaprolactone (mPEG-PCL) missed with a block copolymer of polycaprolactone- $b$-polyethylene glycol with an RGD peptide conjugated (PCL-PEG-RGDfk) forming a treatment known as LKTM (Pan et al. 2018). The formulations were tested in vivo in a thrombosis model in mice. The study showed that those mice treated with LKTM exhibited a significantly shorter tail bleeding time compared to those treated with LK alone or the LK micelles without targeting, hence, suggesting that the novel LKTM had effectively reduced the bleeding risk (Pan et al. 2018).

Other mixed micelle systems reported include the blending of different Pluronic ${ }^{\circledR}$ mixtures (F127 \&P123) for the solubilisation of olmesartan medoxomil (OLM) (El-Gendy et al. 2017). OLM is an antihypertensive drug with poor aqueous solubility which results in low bioavailability (Norwood et al. 2002) OLM was loaded into mixed micelles carriers to overcome this physicochemical barrier. Three drugs: Pluronic ${ }^{\circledR}$ mixture ratios $(1: 40,1: 50$, and 1:60) and various F127:P123 ratios were prepared. The study showed that the mixed micelles were capable of up to $43 \%$ release efficiency compared to $35 \%$ in the drug suspension (El-Gendy et al. 2017).

\section{Dendrimers}

The term dendrimer stems from the Greek 'dendron', meaning tree, accurately reflecting the structure of these repeatedly branched molecules (Crampton and Simanek 2007). The uniqueness of dendrimers stems from their multibranched, three-dimensional architecture, coupled with low polydispersity and great functionality (Sherje et al. 2018). There are many apparent advantages to the utilisation of dendrimers as non-viral vectors for therapeutic indications, as opposed to other nanotechnologies. Their high solubility, greater stability, minimal immunogenicity, and ability to facilitate effective delivery of drug molecules, DNA, and RNAs make them superior to other viral and non-viral counterparts (Mendes et al. 2017). One drawback to dendrimers is that they often possess highly charged exteriors due to their high number of branches at their surface all with their own surface charges; this can often result in either highly cationic or highly anionic nature and, if not fully addressed, can lead to toxicity issues (Jain et al. 2010).

The topology of dendrimers is classified as the focal core, multiple peripheral functional groups, and building blocks with a number of repeating units within the inner core (Jang and Aida 2003). The focal core within a dendrimer has been exploited to host chemical species within, due to the nanoscale environment generated through extensive branching. Second, dendrimers can display various, multiple functional groups on the periphery, allowing for a variety of interactions between other dendrimers and the surrounding molecular environment. Coupled with this, the repeated units permit flexibility within the dendrimer, allowing for the manipulation and encapsulation of drug molecules within (Jang and Aida 2003). The exclusivity of these properties to dendritic molecules gives rise to the possibility for their use as drug delivery systems. The improved efficacy and lower leakage percentages in dendrimers make them 
more suitable in various formulations, including oral, ocular, and transdermal, since the pharmacokinetic parameters for dendrimers are also promising (Yu et al. 2015).

Nitric oxide (NO) is a potent vasodilator, regulator of vascular cell proliferation, and maintains vascular homeostasis. Due to these effects, it can, therefore, play a key role in preventing atherosclerosis (Napoli et al. 2006). Studies have shown that there is potential for NO encapsulation within dendrimers as a targeted therapy for damaged tissue. $\mathrm{Lu}$ et al. (2011) reported that NO release was successful from poly(propylene imine) dendrimers, which shows promise in their potential uses as drug delivery systems ( $\mathrm{Lu}$ et al. 2011). Dendrimers with potential as gene delivery agents for the treatment of CVDs have also been investigated, in an effort to control the upregulation of inflammation in targeted sites within insulted vasculature. Studies have shown that dendrimers carrying DNA plasmids enhanced survivability, stability, and viability of the gene within the nucleus (Gothwal et al. 2015), demonstrating viability as prospective therapeutic agents.

Recently, a dendrimer composed of poly(glutamic acid) and poly(ethylene glycol) (PEG) (Gn-PEG-Gn) was reported for the delivery of nattokinase (NK) (Zhang et al. 2017). NK is a thrombolytic drug, which possessed high safety levels and low side effects, however, extremely sensitive to degradation from the external environment. Hence, careful formulation is required for the drug to be clinically useful. The NK-loaded G3-PEG-G3 dendrimer formed exhibited excellent thrombolytic activity in vitro, and further work is ongoing to elucidate the in vivo potential of this system (Zhang et al. 2017).

Another study reported the development of a poly(amidoamine) (PAMAM) dendrimer-based system as drug carriers for combined delivery of ramipril (RAPL) and hydrochlorothiazide (HCTZ) (Singh et al. 2017). RAPL is an antihypertensive drug and HCTZ is a diuretics drug, both of which are used in the treatment of high blood pressure (de Leeuw and Birkenhäger 1987; Roush and Sica 2016). At $0.8 \%(\mathrm{w} / \mathrm{v})$, dendrimer concentration the RAPL solubility was increased 4.91-fold with amine-terminated dendrimer, whilst the optimal dendrimer for HCTZ solubilisation (3.72fold) was with carboxy-terminated functional groups. The novel formulations resulted in more rapid dissolution compared to the free drugs. Hybrid formulations showed similar dissolution patterns compared to the single drug-loaded dendrimer. The authors concluded that the formulation strategy held promise for application and more work is ongoing to push this technology forward (Singh et al. 2017).

\section{Gel nanoparticles}

The least common on the polymer-based particles are the gelated nanoparticles. These nanoparticles form at with increased polymer concentration into hydrogel matrices into which drugs can be incorporated. These systems allow for a greater retardation of drug release and greater long-term stability (Hamidi et al. 2008).

Regeneration of the ischaemic heart through utilisation of vascular endothelial growth factor (VEGF)-loaded core/shell nanoparticles and their gelation behaviour was monitored to assess vascular cell regeneration (Oh et al. 2010). The core of the gel nanoparticle was constituted from lecithin containing VEGF, with a shell comprising of Pluronic F-127 (poly(ethylene oxide)-poly(propylene oxide)-poly(ethylene oxide) triblock copolymer) (Oh et al. 2010). The temperature-induced gel was formed when the nanoparticles were added to a solution containing propylene glycol monocaprylate at body temperature. Gelated and non-gelated nanoparticles were added to the in vivo sample and functional analysis tests were conducted on the myocardial tissue. The results demonstrated that there was a greater improvement to heart function (cardiac output and ejection fraction) with gelated VEGF-loaded core/shell nanoparticles as compared to non-gelated nanoparticulates (Oh et al. 2010).

\section{Nano-coated stents}

Coronary artery bypass graft $(\mathrm{CABG})$ and percutaneous transluminal coronary angioplasty (PTCA)/percutaneous coronary intervention (PCI) are invasive therapies used commonly within clinical practice to relive blockages in myocardial vasculature. CABG involves major cardiac surgery, whereas PTCA is a non-surgical procedure, whereby an artery is widened using a balloon and, in some instances, is held open using a stent (NICE 2008). The stents used are composed of thin wire mesh, which is used to retain sufficient blood flow through the previously occluded artery, thus preserving its patency (Fig. 4).

The overbearing issue with the use of stents in reperfusion of cardiac tissue leads to a significant risk of restenosis within the vasculature. Restenosis occurs due to overdistention of the diseased vessel, resulting in endothelial disruption, internal elastic laminal fracture, and medical dissection (Bennett 2003). A number of processes contribute to in-stent stenosis including elastic recoil, relocation of axially positioned plaques, and negative remodelling. Neointima formation is the major cause of in-stent

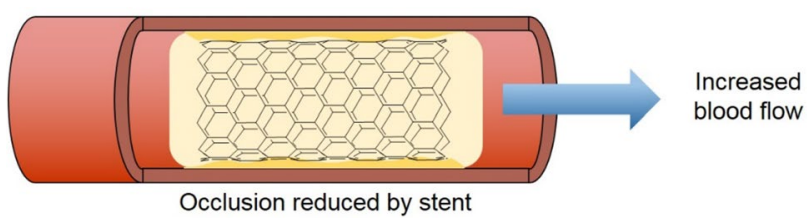

Fig. 4 Use of a stent to widen an occluded blood vessel 
stenosis, whereby platelets, mitogens, and cytokines aggregate. Cell proliferation results and synthesis of the extracellular matrix and collagen lead to the creation of the neointima (Bennett 2003).

A method that can be employed to reduce the incidence of in-stent stenosis is the use of drug-eluting stents (DES), which release the incorporated drug to the locality of its placement. The stents can be coated in various polymers, such as poly(lactic-co-glycolic acid) (PLGA), polyethylene glycol (PEG), and polycaprolactone (PCL) (Acharya et al. 2012). The use of polyhedral oligomeric silsesquioxane poly(carbonate-urea) urethane (POSS-PCU) polymer with attached anti-CD34 antibodies was studied as a possible nanocomposite polymer to coat bare metal stents, in an attempt to improve endothelial revascularisation (Tan et al. 2013). The results of this study indicated that POSS-PCU had advanced biocompatibility and haemo-compatibility in vitro. This is further supported by research into the use of POSS-PCU in the field of heart valve replacements. Synthetic valves made of POSS-PCU nanocomposites have been successfully tested, demonstrating their biocompatibility and biostability against oxidation, hydrolysis, and enzymatic attack in vitro and in vivo (Kidane et al. 2009). However, it has also been demonstrated that the use of drug-eluting stents in animal models led to delayed arterial healing and promoted inflammation at the site of the overlap in the implanted stents. Overlapping paclitaxel-eluting stents (PES) showed a greater degree of fibrin deposition and neointima formation, raising concerns over safety in human subjects, drug toxicity, and further systemic complications arising from sites of overlap (Finn et al. 2005). Use of POSS-PCU as a nanocomposite coating on stents has been taken forward into human trials. The first-in-man trial (FIM) studied the efficacy and long-term implications of non-polymeric drug-coated stents (DCS) in patients with de novo coronary artery disease, with follow-up times of 4 months, 12 months, and 5 years. Notably, at 4 months, there was a significantly lower rate of lumen loss with the DCS than PES. The results demonstrated no inferiority to PES in the rate of lumen loss, a subclass of neointimal hyperplasia (Costa et al. 2016). The use of nano-coatings on stents is being trialled currently in the United States: The COBRA PzF Stenting to Reduce the Duration of Triple Therapy in Patients Treated With Oral Anticoagulation Undergoing Percutaneous Coronary Intervention trial is ongoing and aims to investigate if the COBRA PzF stent with a polyzene-F nano-coating can successfully reduce bleeding compared to DES, through reducing the duration of oral anticoagulation therapy to 14 days, rather than the current 6 months, in patients undergoing PCI (US National Library of Medicine 2018). Polyzene-F is a new generation nano-coating based on cobalt-chromium, designed to be highly biocompatible and easily deliverable (CeloNova Biosciences 2018).
Stents and drug-eluting stents are one of the most established technologies for cardiovascular disease treatment. However, as discussed, there are problems in their usage, and although nanotechnology has been employed in their coating to reduce occurrence of rejection in vivo and restenosis, there is a move away from these technology towards flexible polymer scaffolds.

\section{Nanoparticles for diagnostic imaging}

Nanoparticles also have a place in medicine to fulfil an unmet need in the field of imaging. Nanoparticles offer real potential in medical imaging due to their good bioavailability profiles, versatility of use, and ease of manipulation. Nanoparticles offer adaptable qualities to generate multimodal and multifunctional imaging vehicles with modifiable features, allowing for the integration of contrast materials, ligands, and functional groups (Stendahl and Sinusas 2015). Cardiovascular magnetic resonance (CMR) is a noninvasive method for the detection of inflammation: Due to the pathophysiology of coronary heart disease, inflammation is a major complication of the illness, and early detection through the utilisation of these methods can improve prognosis. CMR relies on the observation of changes in myocardial tissue configuration (Bietenbeck et al. 2015). Gadolinium shows the composition of plaques, including the details of the fibrous cap, necrotic core (Cai et al. 2005), macrophage content, and extent of plaque revascularisation (Kampschulte et al. 2004). Furthermore, contrast-enhanced CMR (ceCMR) can be employed for the recognition of myocardial oedema, necrosis, and fibrosis, using gadolinium or fluorine-19 $\left({ }^{19} \mathrm{~F}\right)$ contrast agents (Friedrich et al. 2009). Healthy myocardium clears the contrast agent rapidly, while damaged tissues retain the dye (Bietenbeck et al. 2015). T1-weighted imaging established CMR imaging as a technique, whereby gadolinium-contrast agent was detected in $>90 \%$ of the myocardium following insult as a result of induced MI in in vivo models, successfully demonstrating the accuracy of CMR as a diagnostic tool (Kim et al. 1996). Such advances in diagnostic imaging will also allow a better patient-centred therapy, by clearer identification of atherosclerotic plaques and the extent of atherosclerosis to guide the dosage of statins required to have a better prophylactic effect, for example.

Gold nanoparticles have also shown great promise in both cardiovascular medicine and oncology imaging. Gold is a noble metal and essentially an inert material which is stable and biocompatible (Thakor et al. 2011). Gold particulates occur in a variety of shapes and sizes, including rods, spheres, cages, stars, crescents, and prisms, with sizes varying from 1 to $500 \mathrm{~nm}$. Gold particles are excreted renally. The elimination rate through glomerular filtration can be modified by 
attaching PEG chains to the nanoparticle surface to increase hydrophilicity and circulation time of the nanoparticle (Varna et al. 2018). Gold nanoparticles can be used in cardiovascular optical imaging: photoacoustic imaging is based on detection of both ultrasound and optical imaging, whereby light is used a source of excitation and ultrasound detects sound waves generated by the target, generating images of optical absorption (Chen et al. 2014).

\section{Future perspectives}

Medical technology and treatments for CVDs, in particular, have significantly advanced in an aim to increase the quality of and prolong life. It is imperative to find better solutions for the treatment of CVD as it is estimated that only $15 \%$ of current guidelines for practice are supported by high-quality evidence (Califf 2016). Better lifestyle, diet, medial intervention, earlier detection, prevention strategies, and improved standard of living have all contributed to better prognoses. A large proportion of all premature deaths under the age of 75, within persons whom are residents of the European Union are due to CVDs. Therefore, it is imperative that solutions are sought quickly to effectively treat this pandemic, with the goal to prevent around $80 \%$ of all cardiovascular diseases through the use of nanotechnologies (Letourneur et al. 2014). As of 2014, a study showed that there were 122 nanomedicines going through clinical development, of which 17 had made it to phase II or III trials (Letourneur et al. 2014). Of those in progress, 70 products were oncology based, 19 for infectious diseases, and only 7 for cardiovascular medicine (Letourneur et al. 2014). This highlights the desperate need for greater research and development in this field. There are a number of current, ongoing trials into the therapeutic use of nanotechnology in cardiovascular medicine, notably, the NanoAthero project, funded by the European Commission. The objectives of this phase I trial include successfully undertaking thrombus imaging and treating vulnerable plaques using novel carriers (European Commission 2017). Further to this, Habib et al. are currently studying the possibility of diagnosing heart failure using breath samples and sensors based on nanomaterials (US National Library of Medicine 2018). Further clinical trials are being conducted in the uses of nanotechnology in drug-eluting stents in comparison to bare metal stents: The research has a participant size of 8000 and thus far has demonstrated the promising results (US National Library of Medicine 2018).

\section{Conclusion}

The evidence presented within this review demonstrates that nanotechnology does hold potential for application in the treatment of CVD, demonstrated by translational clinical trials. With increasing investment into the field of nanotechnology and suitable infrastructure worldwide, it is only a matter of time before nanomedicines, nanomaterial devices, and other related technologies successfully complete the rigorous clinical trial process and come onto the market. A certainty is that nanotechnology holds promise to improve patient health and well-being, and that any progression on the current therapies will positively impact the lives of patients globally. To have the greatest possible impact in medicine, more in vivo studies will need to be conducted and clinical trials commissioned to fully understand the behaviours of nanoparticulates systemically. As the future in therapeutics is becoming more geared towards personalised medicine, nanotechnology may be best placed to achieve this intended goal to tailor treatment to individual disease states. Furthermore, increasing healthcare costs mean that it is imperative to seek alternatives to the existing pharmacological and surgical management, to curb these exponential increases. However, there is substantial evidence to support the concept that nanotechnology is yet to make its full impact to revolutionise medicine.

Open Access This article is distributed under the terms of the Creative Commons Attribution 4.0 International License (http://creativeco mmons.org/licenses/by/4.0/), which permits unrestricted use, distribution, and reproduction in any medium, provided you give appropriate credit to the original author(s) and the source, provide a link to the Creative Commons license, and indicate if changes were made.

\section{References}

Acharya G, Lee C, Lee Y (2012) Optimization of cardiovascular stent against restenosis: factorial design-based statistical analysis of polymer coating conditions. PLoS One 7:e43100

Aisha AFA, Abdulmajid AMS, Ismail Z, Alrokayan SA, Abu-Salah KM (2015) Development of polymeric nanoparticles of Garcinia mangostana xanthones in eudragit RL100/RS100 for anti-colon cancer drug delivery. J Nanomater 16:385 (Article 2015:ID 701979)

Akagi D, Oba M, Koyama H, Nishiyama N, Fukushima S, Miyata T, Nagawa H, Kataoka K (2007) Biocompatible micellar nanovectors achieve efficient gene transfer to vascular lesions without cytotoxicity and thrombus formation. Gene Ther 14:1029-1038

Akbarzadeh A, Rezaei-Sadabady R, Davaran S, Joo S, Zarghami N, Hanifehpour Y, Samiei M, Kouhi M, Nejati-Koshki K (2013) Liposome: classification, preparation, and applications. Nanoscale Res Lett 8:102

Al Meslmani B, Mahmoud G, Bakowsky U (2017) Development of expanded polytetrafluoroethylene cardiovascular graft platform based on immobilization of poly lactic- co -glycolic acid

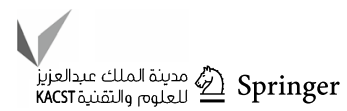


nanoparticles using a wet chemical modification technique. Int J Pharm 529:238-244

Alaarg A, Hamers A, Versloot M, Lobatto M, Mulder WJM, Stroes ESG, Storm G, Metselaar JM (2015) Targeted liposomal drug delivery to inhibit atherosclerotic plaque inflammation. Atherosclerosis. 241:e87

Allijn IE, Czarny SMS, Wang X, Chong SY, Weiler M, da Silva AE, Metselaar JM, Lam CSP, Pastorin G, de Kleijn DPV, Storm G, Wang J-W, Schiffelers RM (2017) Liposome encapsulated berberine treatment attenuates cardiac dysfunction after myocardial infarction. J Control Rel 247:127-133

Banach M, Serban C, Sahebkar A, Mikhailidis D, Ursoniu S, Ray K, Rysz J, Toth P, Muntner P, Mosteoru S, García-García H, Hovingh G, Kastelein J, Serruys P (2015) Impact of statin therapy on coronary plaque composition: a systematic review and metaanalysis of virtual histology intravascular ultrasound studies. BMC Med 13:1-20

Banik BL, Fattahi P, Brown JL (2016) Polymeric nanoparticles: the future of nanomedicine. WIREs Nanomed Nanobiotechnol 8:271-299

Bennett MR (2003) In-stent stenosis: pathology and implications for the development of drug eluting stents. Heart 89:218-224

Bietenbeck M, Florian A, Sechtem U, Yilmaz A (2015) The diagnostic value of iron oxide nanoparticles for imaging of myocardial inflammation-quo vadis? J Cardiovasc Magn Reson 17:1

Binsalamah ZM, Paul A, Prakash S, Shum-Tim D (2012) Nanomedicine in cardiovascular therapy: recent advancements. Expert Rev Cardiovasc Ther 10:805-815

British Heart Foundation (2017) BHF CVD statistics compendium 2017. British Heart Foundation, London, pp 1-123

British Standards Institute (2011) PAS 71:2011: nanoparticles-vocabulary. London: BSI. [Online] https://shop.bsigroup.com/upload/ Shop/Download/Nano/PAS71.pdf. Accessed 19 Apr 2018

Bulbake U, Doppalapudi S, Kommineni N, Khan W (2017) Liposomal formulations in clinical use: an updated review. Pharmaceutics 9:12

Burman A, Mukherjee R, Khatta D, Mullick S, Jaggi M, Singh M, Kumar M, Prusthy D, Gupta P, Praveen R, Singh S (2015) Biocompatible, non-biodegradable, non-toxic polymer useful for nanoparticle pharmaceutical compositions. 8,927,023 B2

Cai J, Hatsukami T, Ferguson M, Kerwin W, Saam T, Chu B, Takaya N, Polissar L, Yuan C (2005) In vivo quantitative measurement of intact fibrous cap and lipid-rich necrotic core size in atherosclerotic carotid plaque: comparison of high-resolution, contrastenhanced magnetic resonance imaging and histology. Circulation 112:3437-3444

Califf R (2016) The future of cardiovascular medicine from the regulatory perspective. J Am Coll Cardiol 68:766-769

CeloNova Biosciences (2018) COBRA PzF nanocoated stent: COBRA PzF stent with polyzene-F nanocoating. [Online]. http://celonova. com/cobra-pzf-nanocoated-stent/. Accessed 18 Apr 2018

Chan JW, Lewis DR, Petersen LK, Moghe PV, Uhrich KE (2016) Amphiphilic macromolecule nanoassemblies suppress smooth muscle cell proliferation and platelet adhesion. Biomaterials 84:219-229

Chen Y, Yeager D, Emelianov S (2014) Photoacoustic imaging for cancer diagnosis and therapy guidance. Cancer theranostic. Elsevier Inc., New York, pp 139-158

Chmielowski RA, Abdelhamid DS, Faig JJ, Petersen KL, Gardner CR, Uhrich KE, Joseph LB, Moghe PV (2017) Athero-inflammatory nanotherapeutics: ferulic acid-based poly(anhydride-ester) nanoparticles attenuate foam cell formation by regulating macrophage lipogenesis and reactive oxygen species generation. Acta Biomater 57:85-94

Chono S, Tauchi Y, Deguchi Y, Morimoto K (2005) Efficient drug delivery to atherosclerotic lesions and the antiatherosclerotic effect by dexamethasone incorporated into liposomes in atherogenic mice. J Drug Target 13:267-276

Costa R, Abizaid A, Mehran R, Schofer J, Schuler G, Hauptmann K, Magalhães M, Parise H, Grube E (2016) Polymer-free biolimus A9-coated stents in the treatment of de novo coronary lesions. JACC Cardiovasc Interv 9:51-64

Cote B, Carlson LJ, Rao DA, Alani AWG (2015) Combinatorial resveratrol and quercetin polymeric micelles mitigate doxorubicin induced cardiotoxicity in vitro and in vivo. J Control Release 213:128-133

Crampton HL, Simanek EE (2007) Dendrimers as drug delivery vehicles: non-covalent interactions of bioactive compounds with dendrimers. Polym Int 56:489-496

de Leeuw PW, Birkenhäger WH (1987) Short- and long-term effects of ramipril in hypertension. Am J Cardiol 59:D79-D82

De Jong WH, Borm PJA (2008) Drug delivery and nanoparticles: applications and hazards. Int J Nanomed 3:133-149

Durán-Lara E, Guzmán L, John A, Fuentes E, Alarcón M, Palomo I, Santos LS (2013) PAMAM dendrimer derivatives as a potential drug for antithrombotic therapy. Eur J Med Chem 69:601-608

El-Gendy MA, El-Assal MIA, Tadros MI, El-Gazayerly ON (2017) Olmesartan medoxomil-loaded mixed micelles: preparation, characterization and in-vitro evaluation. Future J Pharm Sci 3:90-94

Elsabahy M, Wooley K (2012) Design of polymeric nanoparticles for biomedical delivery applications. Chem Soc Rev 41:2545-2561

Eniola-Adefeso O, Heslinga MJ, Porter TM (2012) Design of nano vectors for therapy and imaging of cardiovascular diseases. Methodist DeBakey Cardiovasc J 8:13-17

European Commission (2017) Nanoathero: nanomedicine for targetspecific imaging and treatment of atherosclerosis: development and initial clinical feasibility. [Online]. http://cordis.europa.eu/ project/rcn/106699_en.html. Accessed 19 Apr 2018

Fares AR, El Meshad AN, Kassem MAA (2018) Enhancement of dissolution and oral bioavailability of lacidipine via pluronic P123/ F127 mixed polymeric micelles: formulation, optimization using central composite design and in vivo bioavailability study. Drug Deliv 25:132-142

Finn A, Kolodgie F, Harnek J, Guerrero L, Acampado E, Tefera K, Skorija K, Weber D, Gold H, Virmani R (2005) Differential response of delayed healing and persistent inflammation at sites of overlapping sirolimus- or paclitaxel-eluting stents. Circulation 112:270-278

Fox K, Cowie M, Wood D, Coats A, Gibbs J, Underwood S, Turner R, Poole-Wilson P, Davies S, Sutton G (2001) Coronary artery disease as the cause of incident heart failure in the population. Eur Heart J 22:228-236

Friedrich M, Sechtem U, Schulz-Menger J, Holmvang G, Alakija P, Cooper L, White J, Abdel-Aty H, Gutberlet M, Prasad S, Aletras A, Laissy J, Paterson I, Filipchuk N, Kumar A, Pauschinger M, Liu $\mathrm{P}$ (2009) Cardiovascular magnetic resonance in myocarditis: a JACC white paper. J Am Coll Cardiol 53:1475-1487

Gao Y, Xu P, Chen L, Li Y (2010) Prostaglandin E1 encapsulated into lipid nanoparticles improves its anti-inflammatory effect with low side-effect. Int J Pharm 387:263-271

Gaucher G, Dufresne M, Sant V, Kang N, Maysinger D, Leroux J (2005) Block copolymer micelles: preparation, characterization and application in drug delivery. J Control Release 109:169-188

Geisler T, Langer H, Wydymus M, Gohring K, Zurn C, Bigalke B, Stellos K, May A, Gawaz M (2006) Low response to clopidogrel is associated with cardiovascular outcome after coronary stent implantation. Eur Heart J 27:2420-2425

Giannouli M, Karagkiozaki V, Pappa F, Moutsios I, Gravalidis C, Logothetidis S (2018) Fabrication of quercetin-loaded PLGA nanoparticles via electrohydrodynamic atomization for cardiovascular disease. Mater Today Proc 5:15998-16005 
Gothwal A, Kesharwani P, Gupta U, Khan I, Amin MCIM, Banerjee S, Iyer AK (2015) Dendrimers as an effective nanocarrier in cardiovascular disease. Curr Pharm Des 21:4519-4526

Gupta A (2011) Nanomedicine approaches in vascular disease: a review. Nanomed Nanotechnol Biol Med 7:.763-779

Gupta A, Gupta M (2005) Synthesis and surface engineering of iron oxide nanoparticles for biomedical applications. Biomaterials 26:3995-4021

Hamidi M, Azadi A, Rafiei P (2008) Hydrogel nanoparticles in drug delivery. Adv Drug Deliv Rev 60:1638-1649

Harrington R, Hodgson P, Larsen R (2003) Antiplatelet therapy. Circulation 108:45-47

Hoskins C, Thoo-Lin PK, Cheng WP (2012) A review of comb-shaped amphiphilic polymers for hydrophobic drug solubilisation. Ther Deliv 3:59-79

Hosseini H, Li Y, Kanellakis P, Tay C, Cao A, Tipping P, Bobik A, Toh B-H, Kyaw T (2015) Phosphatidylserine liposomes mimic apoptotic cells to attenuate atherosclerosis by expanding polyreactive IgM producing B1a lymphocytes. Cardiovasc Res 106:443-452

Huang Z, Han Z, Ye B, Dai Z, Shan P, Lu Z, Dai K, Wang C, Huang W (2015) Berberine alleviates cardiac ischemia/reperfusion injury by inhibiting excessive autophagy in cardiomyocytes. Eur J Pharmacol 762:1-10

Immordino M, Dosio F, Cattel L (2006) Stealth liposomes: review of the basic science, rationale, and clinical applications, existing and potential. Int J Nanomed 1:297-315

Jain K, Keharwani P, Gupta U, Jain NK (2010) Dendrimer toxicity: lets meet the challenge. Int J Pharm 394:122-142

Jain PP, Leber R, Nagaraj C, Leitinger G, Lehofer B, Olschewski H, Olschewski A, Prassi R, Marsh LM (2014) Liposomal nanoparticles encapsulating iloprost exhibit enhanced vasodilation in pulmonary arteries. Int J Nanomed 9:3249-3261

Jang W-D, Aida T (2003) Dendritic Physical Gels: Structural Parameters for Gelation with Peptide-Core Dendrimers. Macromolecules 36:8461-8469

Jiang W, Rutherford D, Vuong T, Liu H (2017) Nanomaterials for treating cardiovascular diseases: a review. Bioact Mater 2:185-198

Kagaya H, Oba M, Miura Y, Koyama H, Ishii T, Shimada T, Takato T, Kataoka K, Miyata T (2011) Impact of polyplex micelles installed with cyclic RGD peptide as ligand on gene delivery to vascular lesions. Gene Ther 19:61-69

Kajal A, Kishore L, Kaur N, Gollen R, Singh R (2016) Therapeutic agents for the management of atherosclerosis from herbal sources. Beni Suef Univ J Basic Appl Sci 5:156-169

Kakran M, Sahoo NG, Li L, Judeh (2012) Fabrication of quercetin nanoparticles by anti-solvent precipitation method for enhanced dissolution. Powder Technol 223 7:59-64

Kampschulte A, Ferguson M, Kerwin W, Polissar L, Chu B, Saam T, Hatsukami T, Yuan C (2004) Differentiation of intraplaque versus juxtaluminal hemorrhage/thrombus in advanced human carotid atherosclerotic lesions by in vivo magnetic resonance imaging. Circulation 110:3239-3244

Kidane A, Burriesci G, Edirisinghe M, Ghanbari H, Bonhoeffer P, Seifalian A (2009) A novel nanocomposite polymer for development of synthetic heart valve leaflets. Acta Biomater 5:2409-2417

Kim R, Chen E, Lima J, Judd R (1996) Myocardial Gd-DTPA kinetics determine MRI contrast enhancement and reflect the extent and severity of myocardial injury after acute reperfused infarction. Circulation 94:.3318-3326

Kapil N, Datta YH, Alakbarova N, Bershad E, Selim M, Liebeskind DS, Bachour O, Rao GHR, Divani AA (2017) Antiplatelet and Anticoagulant Therapies for Prevention of Ischemic Stroke. Clin Appl Thrombosis/Hemostasis 23:301-318

Korin N, Kanapathipillai M, Matthews B, Crescente M, Brill A, Mammoto T, Ghosh K, Jurek S, Bencherif S, Bhatta D, Coskun A, Feldman C, Wagner DD, Ingber DE (2012) Shear-activated nanotherapeutics for drug targeting to obstructed blood vessels. Science 10:738-742

Kumar M, Curtis A, Hoskins C (2018) Application of nanoparticle technologies in the combat against anti-microbial resistance. Pharmaceutics 10:11

Kuo C-F, Leon L, Chung EJ, Huang R-T, Sontag TJ, Reardon CA, Getz GS, Tirrell M, Fang Y (2014) Inhibition of atherosclerosis-promoting microRNAs via targeted polyelectrolyte complex micelles. J Mater Chem B 2:8142-8153

Letourneur D, Chauvierre C, Perk J (2014) NanoAthero project: a new way to fight cardiovascular diseases. [Online]. https://www. openaccessgovernment.org/wp-content/uploads/2014/05/INSER M-ebook-plus-edit-web.pdf. Accessed 19 Apr 2018

Levchenko TS, Hartner WC, Torchilin VP (2012) Liposomes in diagnosis a and treatment of cardiovascular disorders. Methodist DeBakey Cardiovasc J 8:36-41

Letchford K, Burt H (2007) A review of the formation and classification of amphiphilic block copolymer nanoparticulate structures: micelles, nanospheres, nanocapsules and polymersomes. Eur J Pharm Biopharm 65:259-69

Lu Y, Sun B, Li C, Schoenfisch M (2011) Structurally diverse nitric oxide-releasing poly(propylene imine) dendrimers. Chem Mater 23:.4227-4233

Luo X, Li J, Guo L, Cheng X, Zhang T, Deng Y (2013) Preparation of berberine hydrochloride long-circulating liposomes by ionophore A23187-mediated $\mathrm{ZnSO}_{4}$ gradient method. Asian J Pharm Sci $8: 261-266$

Ma R, Ma Z-G, Zhen C-L, Shen X, Li S-L, Li L, Zheng Y-F, Dong D-L, Sun Z-J (2017) Design, synthesis and characterization of poly(methacrylic acid-niclosamide) and its effect on arterial function. Mater Sci Eng C 77:352-359

Matoba T, Koga J-I, Nakano K, Egashira K, Tsutsui H (2017) Nanoparticle-mediated drug delivery system for atherosclerotic cardiovascular disease. J Cardiol 70:206-211

Mendes LP, Pan J, Torchilin VP (2017) Dendrimers as nanocarriers for nucleic acid and drug delivery in cancer therapy. Molecules 22:E1401

Mitchell P, Marle D, Donkor A, Shote A, McDonagh T, Hardman S, Dargie H, Cleland J (2015) National heart failure audit. National Institute for Cardiovascular Outcomes Research, London, pp $1-72$

Mourya VK, Inamdar N, Nawale RB, Kulthe SS (2011) Polymeric micelles: general considerations and their application. Indian $\mathrm{J}$ Pharm Ed Res 45:128-138

Mukhametova LI, Zakharyan EM, Karakhanov EA, Gershkovich KB, Varfolomeyev SD (2017) Thrombolytic and fibrinogenolytic properties of bioconjugate streptokinase-polyamidoamine dendrimers in vitro. Thromb Res 154:50-52

Murohara T, Margiotta J, Phillips LM, Paulson JC, DeFrees S, Zalipsky S, Guo LS, Lefer AM (1995) Cardioprotection by liposome-conjugated sialyl Lewisx-oligosaccharide in myocardial ischaemia and reperfusion injury. Cardiovasc Res 30:965-974

Nahar K, Absar S, Gupta N, Kotamraju VR, McMurtry IF, Oka M, Komatsu M, Nozik-Grayck E, Ahsan F (2014) Peptide-coated liposomal fasudil enhances site specific vasodilation in pulmonary arterial hypertension. Mol Pharm 11:4374-4384

Napoli C, de Nigris F, Williams-Ignarro S, Pignalosa O, Sica V, Ignarro LJ (2006) Nitric oxide and atherosclerosis: an update. Nitric Oxide 15:265-79

National Institute for Health and Clinical Excellence (NICE) (2008) Guidance on the use of coronary artery stents. NICE guidelines (TA71)

National Institute for Health and Clinical Excellence (NICE) (2010) Chronic heart failure in adults: management. Guideline NICE (CG108) 
National Institute for Health and Clinical Excellence (NICE) (2013) Myocardial infarction: cardiac rehabilitation and prevention of further cardiovascular disease. NICE guideline (CG172)

Norwood D, Branch E, Smith B, Honeywell M (2002) Olmesartan medoxomil for hypertension: a clinical review. P\&T Drug Forecast 27:611-618

Oh K, Song J, Yoon S, Park Y, Kim D, Yuk S (2010) Temperatureinduced gel formation of core/shell nanoparticles for the regeneration of ischemic heart. J Control Release 146:207-211

Önyüksel H, Ikezaki H, Patel M, Gao X-p, Rubinstein I (1999) A novel formulation of VIP in sterically stabilized micelles amplifies vasodilation in vivo. Pharm Res 16:155-160

Palekar RU, Myerson JW, Schlesinger PH, Sadler JE, Pan H, Wickline SA (2013a) Thrombine targeted liposomes establish a sustained localized anticlotting barrier against acute thrombosis. Mol Pharm 10:4168-4175

Palekar RU, Myerson JW, Schlesinger PH, Pan H, Wickline SA (2013b) Thrombin-inhibiting liposomes as a targeted therapeutic for the treatment of acute thrombosis. FASEB J 27:1

Palekar RU, Jallouk AP, Lanza GM, Pan H, Wickline SA (2015) Molecular imaging of atherosclerosis with nanoparticle-based fluorinated MRI contrast agents. Nanomed (Lond) 10:11

Pan Y, Wang X, Yin Z (2018) Synthesis and evaluation of cationic polymeric micelles as carriers of lumbrokinase for targeted thrombolysis. Asian J Pharm Sci (In press)

Pang X, Lu Z, Du H, Yang X, Zhai G (2014) Hyaluronic acid-quercetin conjugate micelles: synthesis, characterization, in vitro and in vivo evaluation. Colloid Surface B Biointerface 123:778-786

Patel D, Bailey S (2007) Nanotechnology in cardiovascular medicine. Catheter Cardiovasc Interv 69:643-654

Patil M, Mehta D, Guvva S (2008) Future impact of nanotechnology on medicine and dentistry. J Indian Soc Periodontol 12:34-40

Pazzini C, Marcato PD, Prado LB, Alessio AM, Höehr NF, Montalvão S, Paixão D, Durán N, Annichino-Bizzacchi JM (2015) Polymeric nanoparticles of enoxaparin as a delivery system: in vivo evaluation in normal rats and in a venous thrombosis rat model. J Nanosci Nanotechnol 15:4837-4843

Ponikowski P, Voors A, Anker S, Bueno H, Cleland J, Coats A, Falk V, González-Juanatey J, Harjola V, Jankowska E, Jessup M, Linde C, Nihoyannopoulos P, Parissis J, Pieske B, Riley J, Rosano G, Ruilope L, Ruschitzka F, Rutten F, van der Meer P (2016) 2016 ESC guidelines for the diagnosis and treatment of acute and chronic heart failure. Eur Heart J 37:2129-2200

Qin H, Zhao Y, Zhang J, Pan X, Yang S, Xing D (2016) Inflammationtargeted gold nanorods for intravascular photoacoustic imaging detection of matrix metalloproteinase-2 (MMP 2) in atherosclerotic plaques. Nanomed Nanotechnol Biol Med 12:1765-1774

Roush GC, Sica DA (2016) Diuretics for hypertension: a review and update. Am J Hypertens 29:1130-1137

Ruiz-Esparza G, Flores-Arredondo J, Segura-Ibarra V, Torre-Amione G, Ferrari M, Blanco E, Serda R (2013) The physiology of cardiovascular disease and innovative liposomal platforms for therapy. Int J Nanomed 8:629-640

Sahoo S, Labhasetwar V (2003) Nanotech approaches to drug delivery and imaging. Drug Discov Today 8:1112-1120

Sailor G, Seth AK, Parmer G, Chauhan S, Javia A (2015) Formulation and in vitro evaluation of berberine containing liposome optimized by $3^{2}$ full factorial designs. J Appl Pharm Sci 5:023-028

Sanchez-Gaytan BL, Fay F, Lobatto ME, Tang J, Ouimet M, Kim YT, van der Staay SEM, van Rijs SM, Priem B, Zhang L, Fisher EA, Moore KJ, Langer R, Fayad ZA, Mulder WJM (2015) HDLmimetic PLGA nanoparticle to target atherosclerosis plaque. Macrophages Bioconj Chem 26:443-451

Sanna V, Pala N, Sechi M (2014) Targeted therapy using nanotechnology: focus on cancer. Int J Nanomed 9:467-483
Sherje AP, Jadhav M, Dravyakar BR, Kadam D (2018) Dendrimers: a versatile nanocarrier for drug delivery and targeting. Int J Pharm 548:7070-7720

Siddalingappa B, Benson HAE, Brown DH, Batty KT, Chen Y (2015) Stabilization of resveratrol in blood circulation by conjugation to $\mathrm{mPEG}$ and mPEG-PLA polymers: investigation of conjugate linker and polymer composition on stability, metabolism, antioxidant activity and pharmacokinetic profile. PLoS One 10:e0118824

Singh MK, Pooja D, Kulhari H, Jain SK, Sistla R, Chauhan AS (2017) Poly(amidoamine) dendrimer-mediated hybrid formulation for combination therapy of ramipril and hydrochlorothiazide. Eur J Pharm Sci 96:84-92

Singla P, Singh O, Chabba S, Mahajan RK (2018) Pluronic-SAILs (surface active ionic liquids) mixed micelles as efficient hydrophobic quercetin drug carriers. J Mol Liq 249:294-303

Stendahl J, Sinusas A (2015) Nanoparticles for cardiovascular imaging and therapeutic delivery, part 1: compositions and features. J Nucl Med 56:1469-1475

Tan A, Farhatnia Y, Goh DGN, de Mel A, Lim J, Teoh S, Malkovskiy A, Chawla R, Rajadas J, Cousins B, Hamblin M, Alavijeh M, Seifalian A (2013) Surface modification of a polyhedral oligomeric silsesquioxane poly(carbonate-urea) urethane (POSS-PCU) nanocomposite polymer as a stent coating for enhanced capture of endothelial progenitor cells. Biointerphases 8:.23

Tefas LR, Tomata I, Achim M, Vlase L (2015) Development and optimization of quercetin loaded PLGA nanoparticles by experimental design. Clujul Med 88:214-223

Thakor A, Jokerst J, Zavaleta C, Massoud T, Gambhir S (2011) Gold nanoparticles: a revival in precious metal administration to patients. Nano Lett 11:4029-4036

Tomalia DA, Reyna LA, Svenson S (2007) Dendrimers as multipurpose nanodevices for oncology drug delivery and diagnostic imaging. Biochem Soc Trans 35:61-67

Trinh H, Joseph M, Cholkar K, Mitra R, Mitra A (2017) Nanomicelles in diagnosis and drug delivery. In: Mitra A, Cholkar K, Mandal A (eds) Emerging nanotechnologies for diagnostics, drug delivery and medical devices. Elsevier, Amsterdam, Netherlands, pp $45-48$

US National Library of Medicine (2018) Randomized trial of COBRA $\mathrm{PzF}$ stenting to reduce duration of triple therapy. [Online]. https ://clinicaltrials.gov/ct2/show/NCT02594501?term=COBRA + REDUCE\&rank=1. Accessed 18 Apr 2018

Varna M, Xuan H, Fort E (2018) Gold nanoparticles in cardiovascular imaging. Wiley Interdiscip Rev Nanomed Nanobiotechnol 10:1-19

Wan TC, Tosh DK, Du L, Gizewski ET, Jacobson KA, Auchampach JA (2011) Polyamidoamine (PAMAM) dendrimer conjugate specifically activates the $\mathrm{A} 3$ adenosine receptor to improve postischemic/reperfusion function in isolated mouse hearts. BMC Pharmacol 11:11

Wang KY, Tuli L, Cooper E, Wang N, Liu D (2013) Recombinant protein production of earthworm lumbrokinase for potential antithrombic application. Evid Based Complement Altern Med 2013:783971

Weaver J (2013) PLOS Biology 11(4):e1001533

World Health Organization (WHO) (2017) Cardiovascular diseases (CVDs). [Online]. http://www.who.int/mediacentre/factsheets/ fs317/en/. Accessed 18 Apr 2018

Wu T, Chen X, Wang Y, Xiao H, Peng Y, Lin L, Xia W, Long M, Tao J, Shuai X (2018) Aortic plaque-targeted andrographolide delivery with oxidation-sensitive micelle effectively treats atherosclerosis via simultaneous ROS capture and anti-inflammation. Nanomed Nanotechnol Biol Med 14:2215-2226 
Yu M, Jie X, Xu L, Chen C, Shen W, Cao Y, Lian G, Qi R (2015) Recent advances in dendrimer research for cardiovascular diseases. Biomacromolecules 16:2588-2598

Zhai Y, Guo S, Liu C, Yang C, Dou J, Li L, Zhai G (2013) Preparation and in vitro evaluation of apigenin-loaded polymeric micelles. Colloid Surface A Phys Eng Asp 429:24-30. https:// doi.org/10.1016/j.colsurfa.2013.03.051

Zhang Y, Yang S, Li M, Iqbal J, Bourantas CV, Mi Q, Yu Y, Li J, Zhao S, Tian N, Chen S (2014) Berberine attenuates adverse left ventricular remodeling and cardiac dysfunction after acute myocardial infarction in rats: role of autophagy. Clin Exp Pharmacol Physiol 41:995-1002

Zhang S-F, Gao C, Lü S, He J, Liu M, Wu C, Liu Y, Zhang X, Liu Z (2017) Synthesis of PEGylated polyglutamic acid peptide dendrimer and its application in dissolving thrombus. Colloid Surface B Biointerface 159:284-292

Zhang M, Li C, Zhou D, Ding C, Jin Y, Tian Q, Meng X, Pu K, Zhu Y (2018) Cyclic RGD functionalized liposomes encapsulating urokinase for thrombolysis. Acta Biomater 70:227-236

Publisher's Note Springer Nature remains neutral with regard to jurisdictional claims in published maps and institutional affiliations. 